\title{
How can fertility counseling be implemented for every newly diagnosed pediatric patient facing gonadotoxic treatment?-A single-center experience
}

\author{
Anke Barnbrock ${ }^{1,2} \cdot$ Emilia Salzmann-Manrique ${ }^{1} \cdot$ Nicole Sänger $^{3} \cdot$ Henning Fiegel $^{4} \cdot$ Falk Ochsendorf $^{5}$. \\ Thomas Klingebiel $^{1,2} \cdot$ Peter Bader $^{1} \cdot$ Andrea Jarisch $^{1}$
}

Received: 7 March 2021 / Accepted: 17 August 2021 / Published online: 18 September 2021

(C) The Author(s) 2021

\begin{abstract}
Since the survival rates of pediatric patients undergoing cancer treatment or hematopoietic stem cell transplantation (HSCT) have increased rapidly in recent decades, the late effects of treatment are now an important focus of patient care. Access to fertility preservation (FP) procedures as well as their financing differs considerably across Europe. However, some countries in Europe have recently changed the legal basis for financing FP procedures; therefore, the implementation of structures is mandatory to give patients access to FP. In this prospective cohort study, we characterized the process for establishing pediatric fertility counseling, including the development of an in-house standard procedure for recommendations regarding FP with potentially gonadotoxic treatment and valuating data from all FP counseling sessions. All data concerning patient characteristics (pubertal status, disease group) and recommendation of FP measures were prospectively collected and adoption of FP measures analyzed. Prior to the establishment of a structured process for FP in our pediatric oncology and stem cell transplantation center, there was no standardized FP counseling. We demonstrate that with the establishment of an inhouse standard procedure, it is possible to give consistent yet individualized FP counseling to approximately $90 \%$ of our patients facing gonadotoxic treatment, counseling over 200 patients between 2017 and 2019. This pilot study could potentially be adapted in other pediatric hematology, oncology, and stem cell transplantation centers to allow a more standardized handling of FP counseling for all patients facing gonadotoxic treatment.
\end{abstract}

Keywords Fertility counseling $\cdot$ Pediatric oncology $\cdot$ Pediatric stem cell transplantation $\cdot$ Indication for fertility preservation

Anke Barnbrock

anke.barnbrock@kgu.de

1 Division for Stem Cell Transplantation and Immunology, Department for Children and Adolescents, University Hospital, Goethe University, Frankfurt/Main, Germany

2 Division for Pediatric Hematology and Oncology, Department for Children and Adolescents, University Hospital, Goethe University, Theodor-Stern-Kai 7, 60596 Frankfurt/Main, Germany

3 Division for Gynecological Endocrinology and Reproductive Medicine, Department for Gynecology and Obstetrics, University Hospital Bonn, Bonn, Germany

4 Division for Pediatric Surgery and Pediatric Urology, Department for Surgery, University Hospital, Goethe University, Frankfurt/ Main, Germany

5 Division for Andrology, Department for Dermatology, Venerology and Allergology, University Hospital, Goethe University, Frankfurt/ Main, Germany

\begin{abstract}
Abbreviations
ASCO American Society of Clinical Oncology

ASRM American Society for Reproductive Medicine

AWMF Arbeitsgemeinschaft der Wissenschaftlichen Medizinischen Fachgesellschaften e.V.

EBMT European Society for Blood and Marrow Transplantation
\end{abstract}

\section{Introduction}

With the immense progress in therapeutic regimens in pediatric oncology, the survival rates have increased up to $80 \%$ [1]. Therefore, broader indications for various diseases in the malignant and especially the nonmalignant settings have led to an increasing number of patients treated worldwide [2] and to a better overall long-term outcome. The possibility of family planning and having biological children is crucial for long-term survivors, and infertility drastically downgrades quality of life [3]. 
The risk of infertility due to chemo- or radiotherapy depends on the underlying disease, age, sex, other comorbidities, and the choice and dosage of chemotherapeutics, as well as the determination of the radiation field $[4,5]$. For patients undergoing HSCT, gonadotoxicity is mainly associated with alkylating agents (especially busulfan) and total body irradiation (12 Gy) and the overall risk of infertility after HSCT is 70$80 \%$ [6]. Female patients who are exposed to gonadotoxic treatment experience subfertility with a diminished ovarian reserve at a younger age than the general female population [6].

\section{Current practice}

The current practice of FP counseling and the performance of FP procedures differ among European countries according to national recommendations, local logistics, technical experience and the assumption of costs by the national insurance system. In France and Israel, FP composes part of national law. In 2012, Nordic countries recommended counseling and the possibility of FP procedures for all patients at risk of fertility injury due to oncology treatment, including HSCT. This program is financed by the national insurance system [7]. In Germany and Switzerland, there has been no assumption of costs by the national insurance system until now. However, both countries recently changed the legal basis for financing FP procedures [8], but especially in Germany, coverage of the costs for patients below 18 years of age is still unclear, although the implementation of FP procedures in this age group was recommended by German AWMF guidelines as early as 2015 [9].

FP counseling is a difficult task for physicians and demands a sensitive approach regarding the ethnic and cultural background of the families as well as the maturity and age of the patient. In particular, adolescents should be addressed directly, as they usually wish to be included in the decision-making process [10]. For children and adolescents, it is important to integrate developmentally appropriate patients in the decision-making process by using tools that enable decision-making and assent in children [11]. In addition to the shock of a newly diagnosed malignant disease or the naming of side effects during a pre-HSCT interview, the risk of infertility can be further devastating news and should be taken into consideration [7]. FP counseling helps in devising a strategy to address the problem in the best way and might provide an option for future fertility.

FP counseling should include:

1. Risk assessment of infertility according to the patient's age, treatment/conditioning regimen, and known comorbidities with possible impacts on fertility.

2. Presentation of FP techniques eligible for the counselled patient and their established or experimental characteristics.

3. Rational and individualized decision-making with patients/caregivers taking into account the risk of delaying treatment, the risk of transferring malignant cells with gonadal tissue, and the risk of side effects from the FP procedure itself.

\section{Options for FP in boys and girls}

There are some well-established and experimental treatment options available for FP. Cryopreservation of sperm is a standard procedure [12], while cryopreservation of immature testicular tissue remains experimental and should only be performed within study protocols to ensure ongoing research in this field [13]. Recently, the grafting of autologous premature testes of monkeys resulted in adult spermiogenesis and, finally, in the birth of a healthy baby monkey [14]. These results are promising for preserving fertility in prepubertal boys. For postpubertal female patients, the cryopreservation of unfertilized eggs has been an established method of FP for years [15]. If it is not possible to delay the start of therapy for this procedure, cryopreservation of ovarian tissue for later retransplantation is recommended [16]. The American Society for Reproductive Medicine (ASRM) recently concluded that ovarian tissue cryopreservation is an acceptable FP technique and should no longer be considered experimental [17]. In prepubertal female patients, it is the only method for preserving fertility. Some reports have already described children born after retransplantation of cryopreserved prepubertal ovarian tissues [18, 19]. In FP that involves cryopreservation of tissue, the risk of the reintroduction of malignant cells with the cryopreserved tissue has to be discussed with the patients and their families [20]. The risk should be considered low in some cases of solid tumors and Hodgkin lymphomas, intermediate in cases of non-Hodgkin lymphomas and some metastasized solid tumors such as Ewing's sarcoma or rhabdomyosarcomas. This risk is highest in cases of neuroblastoma and leukemia, primarily acute lymphoblastic leukemia, which was shown in post-mortem tissue analyses as well as in FP cryopreserved ovarian tissue [21, 22].

\section{Objective}

We describe the process of the implementation of fertility counseling and our experience in the Division of Pediatric Oncology and Stem Cell Transplantation using an inhouse standard procedure established by our team. Before we implemented structured fertility counseling in our center, the risk of gonadotoxic side effects was addressed in the initial interview about the disease and the individualized therapy options with the patient and the family. The counseling did not follow a standard format and was not conducted in a separate interview. The aim of this prospective cohort study was to analyze the demographic characteristics of counseled patients, the percentage of FP recommendation and their adoption by specific 
patients' cohorts (female vs male, pre- vs post-pubertal, nonmalignant vs malignant patient cohort).

Notably, the implementation of a structure for counseling and assessing the performance of FP took place at a time while FP was not yet covered by the German health insurance system. The inability to cover costs and therefore low staffing levels are some reasons why FP counseling has not yet been established in all pediatric units dealing with gonadotoxic treatment.

\section{Materials and methods}

\section{Patients}

This is a prospective cohort study analyzing data collected in a single center with the start of implementation of fertility counseling. From January 2017 to February 2019, we counseled patients with a newly diagnosed malignant disease or who were undergoing HSCT. In our center, we usually see patients $0-18$ years old. Nevertheless, we include young adults as part of the CAYA project (care of adolescents and young adults) for oncological treatment. The majority of patients in this study were less than 18 years old, the oldest 22 years at the most.

As part of the initial work-up, all patients underwent a standardized procedure for examination of pubertal status, including clinical examination, abdominal and, if applicable, testicular sonography and hormone measurements (female patients: LH, FSH, estrogen, anti-mullerian hormone; male patients: LH, FSH, testosterone, inhibin B, sex hormone-binding globulin). The Tanner stages, and in boys' testis volume, were used to determine the clinical stage of puberty. Tanner stages I-II were considered pre-pubertal, Tanner IV-V post-pubertal (including testis volume greater $10 \mathrm{ml}$ clinically and by ultrasound). The clinical findings were compared with the results of the hormone levels. Patients with Tanner stage III could be assigned to either the prepubertal or post-pubertal group based on the hormone values. If this was not possible, they were designated as peri-pubertal. All data were collected as part of the initial work-up and documented in the chart. Following informed consent by the caregivers and the patient, the clinical findings, the counseling and the FP recommendation were documented in a database. Prior to implementation of FP counseling, an ethical approval was obtained for this FP database (see below). The data for the present analysis were taken from this database and the medical files.

\section{In-house standard procedure for fertility counseling and preservation}

Before the structured fertility counseling service was established, the team decided to define a uniform recommendation guideline (in-house standard procedure) so that the vast majority of patients would receive counseling based on international recommendations as well as national, local and individual conditions.

With the medical team, we reviewed recent guidelines based on those of the German, Austrian and Swiss Societies of Pediatric Oncology and Hematology [9], the German Society for Gynecology and Obstetrics, the German Society for Urology and German Society for Reproductive Medicine [23], the American Society of Clinical Oncology [24], and the European Society for Blood and Marrow Transplantation [25] in detail. A list of indications for FP procedures was established for all tumor entities and was used as the inhouse standard procedure for the counseling process at our institution (Supplementary Table 1). This list defines a treatment-related risk score for fertility impairment for each tumor entity according to the above mentioned guidelines [9, 23-25], and a time window within FP had to be implemented to avoid a delay initiating therapy and the risk of contamination of the tissue due to the primary disease and metastatic status at time of diagnosis.

For each patient, we discussed the risk of the FP procedures regarding the general patient condition.

Especially for very young children with a low gonadotoxic risk (for example hepatoblastoma and some nephroblastoma), we did not recommend experimental techniques. For all systemic malignancies (e.g., leukemias) treated with allo-HSCT, we do not recommend tissue cryopreservation at the moment despite the high gonadotoxic risk of the treatment for the risk of reinducing malignant cells with retransplantation of the tissue [20] (see the "Discussion"). For patients with solid tumors, we recommended considering fertility-preserving methods for all tumors with an intermediate risk of gonadotoxicity (50-70\% risk of infertility) [9], such as osteosarcoma and Ewing's sarcoma, unless there was evidence of multiple metastases. In case of intermediate risk of treatment for non-metastasized tumors, the potential of the FP procedure had to be weighed against the potential risk of damaging gonadal tissue through the procedure itself. Cryopreservation procedures were recommended for all patients with nonmalignant diseases treated with HSCT.

Fertility counseling was offered to the parents of all children and adolescents undergoing chemotherapy or HSCT independent of whether FP was recommended or planned to be performed. One devoted person of the oncology/ transplantation team did the counseling, independently from the general informative interview on diagnosis and treatment to ensure the possibility of concentrating solely on the fertility aspect of the diagnosis and treatment plan. The counseling lasted 30-60 min and included a face-toface interview with the caregivers and the patients. In all patients except for those less 6 years, we aimed to give age at least general information on the fertility aspect of their treatment. With the adolescent patients, we discussed the gonadotoxic risk and FP measures in detail. We also offered 
written material to further explore the topic (brochures designed by the German Society of Pediatric Oncology and by our Department of Reproductive Medicine).

To perform the FP procedures, we created a multidisciplinary team composed of members of the Division of Pediatric oncology/hematology and stem cell transplantation, Gynecology/Reproductive Medicine, Andrology, Pediatric Surgery and Psychology/ psychosocial team.

In cases of invasive procedures such as ovarian or testicular tissue cryopreservation, we aimed to time these operations with other procedures, such as the implantation of a tunneled central line, because of the risk of side effects owed to anesthesia and the psychological impact of undergoing multiple surgery. In some cases, the invasive fertility-preserving procedures were performed independently from other operations (for example, if the patient already had a central line when being transferred to our department). However, we usually attempted to avoid this due to the experimental nature of the FP procedures for young patients.

\section{Statistical analysis}

Data analysis was performed with $\mathrm{R}$ version 3.6.1 (foundation for statistical computing, Vienna, Austria). All tests were twosided with a significance level of 0.05 .

Descriptive statistics for categorical data are presented as absolute frequencies and percentages, and those for continuous data are presented as the median, interquartile range (IQR), maximum and minimum. Fisher's exact test was used for comparisons between groups with a two-sided ClopperPearson $95 \%$ confidence interval (CI).

We received approval from the Ethics Committee of the University Hospital Frankfurt/Main (Reg. No. 239/17).

\section{Results}

In total, we counseled 202 patients from the Division of Pediatric Hematology and Oncology (152 patients) and the Division of Pediatric Stem Cell Transplantation (50 patients) between January 2017 and February 2019. In this period, 199 new patients were admitted to our oncology department with newly diagnosed oncological diseases (Fig. 1). Of these, 170 were scheduled to receive either chemotherapy, including auto-HSCT, or radiotherapy as part of their treatment plan. A total of $89.4 \%(N=152)$ of the 170 oncological patients received fertility counseling within the first days of diagnosis, including detailed information regarding the gonadotoxicity of their treatment, and we offered FP procedures if indicated. In the same manner, we counseled 50 patients who were scheduled to receive allo-HSCT. Usually, the counseling lasted 30-60 min and was performed independently from the counseling regarding diagnosis and treatment planning (Fig. 1).

In the total cohort of 202 counselled patients, $41 \%(N=$ $82)$ were girls, and 59\% $(N=120)$ were boys. Fifty-nine percent of the counseled girls $(N=48)$ and $69 \%(N=83)$ of the boys were prepubertal. Only one female and three male patients could not be determined as being either pre- or postpubertal (Table 1). Table 1 shows the demographic data of the patients regarding sex, age group, disease entities and pubertal status. In our cohort, $59 \%$ of the counseled patients were boys; this disparity was especially striking in the leukemia group.

Regarding diagnosis, $35 \%$ of the patients presented with malignant solid tumors, while $34 \%$ were diagnosed with acute leukemias. Lymphoma was found in $13 \%$ of the patients, and myelodysplastic syndrome was found in $3 \%$. Nonmalignant diseases requiring HSCT, such as sickle cell disease, thalassemia major or congenital immunodeficiency syndromes were the underlying medical condition in $14 \%$ of patients.

The results of the fertility counseling process are shown in Figs. 2 and 3. Figure 2 demonstrates that, as expected, the recommendations for fertility-preserving measures depended on pubertal level and sex. We observed a statistically significant increase in FP recommendations between the age groups from prepubertal $(20 \% ; N=26 ; 95 \%$ CI $13-28 \%)$ to postpubertal status $(59 \% ; N=39 ; 95 \%$ CI $46-71 \%)$ $(P<0.001)$ for the whole cohort (Fig. 2A).

Looking at the pubertal subgroups, both the prepubertal and postpubertal groups demonstrated statistically significant differences between the female and male subgroups concerning FP recommendations (Fig. 2B and C). In the prepubertal group, only $33 \%(N=16 ; 95 \%$ CI $20-48 \%)$ of the female patients did receive a recommendation for an invasive procedure, and even less, $12 \%(N=10 ; 95 \%$ CI $6-21 \%)$ of the prepubertal male patients were counseled likewise $(P=0.005)$. In the postpubertal group, this rate of recommendation was $45 \%(N=15 ; 95 \%$ CI $28-64 \%)$ vs. $73 \%(N=24 ; 95 \%$ CI 54 $87 \%)(P=0.044)$ for female and male patients, respectively, thus half of the postpubertal female patients and over two thirds of the postpubertal male patients received a recommendation for a FP procedure

In addition, in the female group, there were no statistically significant differences in the number of patients with no recommendation for a FP procedure between the pre- and postpubertal subgroups ( $67 \%$ vs. $55 \% ; P=0.069)$, but in the male group, the FP recommendations depended strongly on the pubertal status ( $88 \%$ vs. $27 \%$; $P<0.001)$.

The procedures recommended for postpubertal female patients were almost exclusively ovarian tissue cryopreservation. Only one postpubertal female patient underwent hormonal stimulation for oocyte cryopreservation and succeeded in storing unfertilized oocytes for later in vitro fertilization (see the "Discussion" section). 


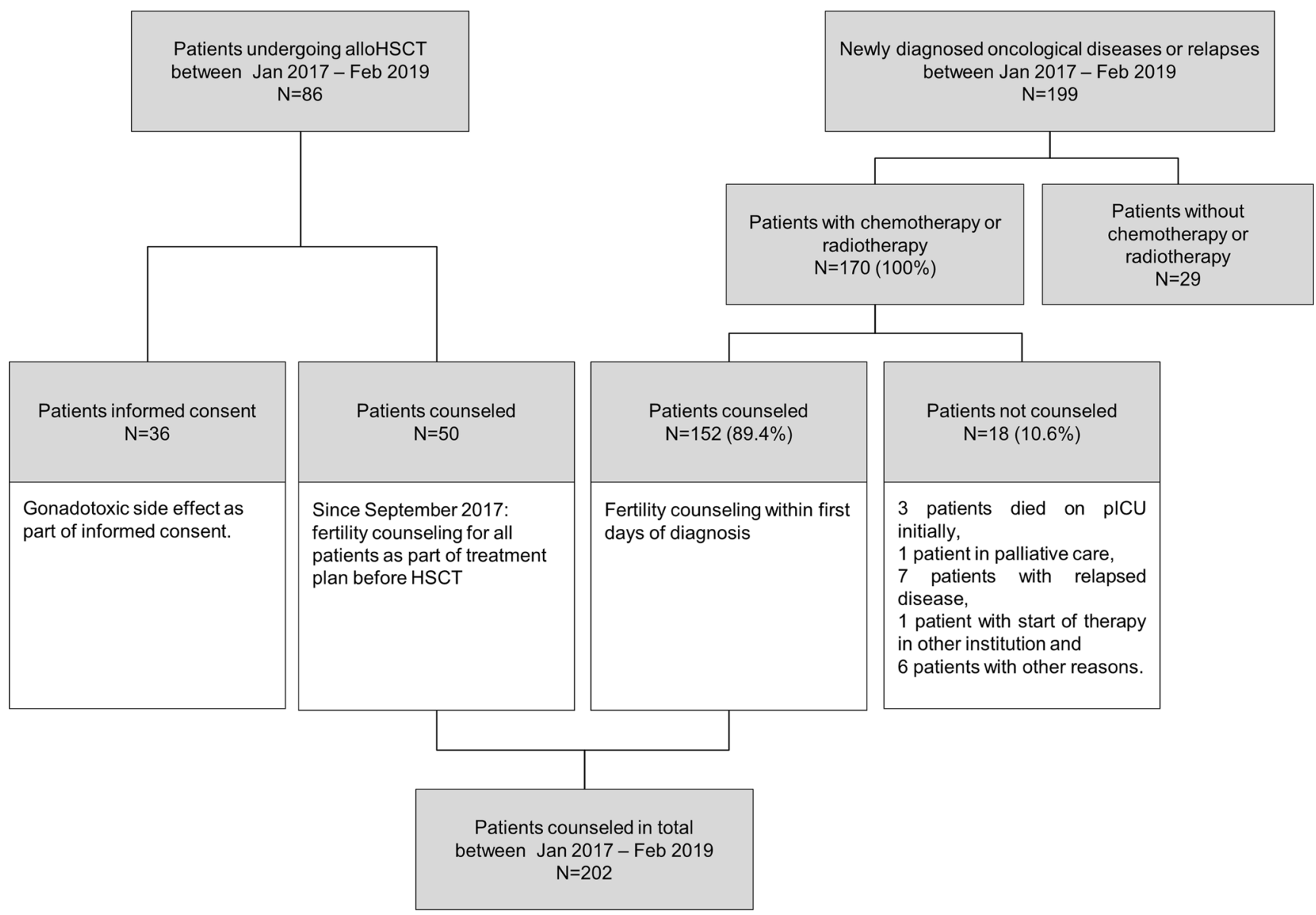

Fig. 1 Analysis of patients receiving fertility counseling in our institution between January 2017 and May 2018. Note: Starting in September 2017, patients planned for allo-HSCT received detailed fertility counseling separately from the general informed consent for allo-HSCT

Figure 3 summarizes the outcome of the counseling process in regard to sex, pubertal stage, and malignant versus nonmalignant disease. In the malignant cohort, we recommended an FP procedure for $25 \%$ of the prepubertal female patients, but only $7.5 \%$ of the patients and their families agreed to it. In the nonmalignant cohort, we advised $75 \%$ of the patients to undergo an invasive procedure according to our in-house standard procedure, and $62.5 \%$ agreed to it (Fig. 3A). In the postpubertal female cohort, we recommended an FP procedure to $36 \%$ of the malignant cohort, and half of the patients followed our recommendation. In the nonmalignant cohort, we counseled $100 \%$ of the patients to undergo a procedure, and $80 \%$ followed our recommendation (Fig. 3C). In the male cohort, the difference between pre- and postpubertal patients and between malignant and nonmalignant disease was even more pronounced. In the prepubertal cohort of patients with malignant diseases, we did not recommend a fertility-preserving measure for $99 \%$, while we recommended an invasive procedure for $100 \%$ of the nonmalignant cohort, and only $25 \%$ of patients did not follow our recommendation (Fig. 3B). We offered sperm cryopreservation to the majority of postpubertal male patients. While $100 \%$ of the nonmalignant cohort followed our recommendation, only $27 \%$ of the malignant cohort did so, mainly for personal reasons (Fig. 3D). For 9 patients, we did not recommend sperm cryopreservation. Two patients had already started chemotherapy at other institutions, 5 patients had a relapse of their disease (4 leukemia, 1 lymphoma), had already started chemotherapy or received radiation therapy of the testis and had come to our institution for SCT, and two patients were in a poor overall medical condition at the time of counseling. The results of the counseling process for the age subgroups are shown in Supplementary Figure 1 (female patients) and Supplementary Figure 2 (male patients).

No patient in the 11-13 year age group, $52 \%$ of the patients in the 14-16 year age group, and 25\% of the 17-22 year age group underwent sperm cryopreservation. For one patient, the clinical status worsened; thus, sperm cryopreservation was no longer feasible. In another patient, the organization of cryopreservation would have resulted in a significant delay in the start of treatment. Six patients did not want to undergo this procedure for personal reasons, and for 3 patients, the quality of the sperm was not suitable for cryopreservation. Only one patient underwent testicular biopsy to cryopreserve the sperm. 
Table 1 Patient characteristics and epidemiologic data. Data shown for female and male patients regarding age groups, disease entity, and pubertal status

\begin{tabular}{|c|c|c|c|c|}
\hline & Total & Girls & Boys & $P$ value \\
\hline N (\%) & $202(100)$ & $82(41)$ & $120(59)$ & \\
\hline FP counselling date & & & & 1 \\
\hline Median & Feb 2018 & April 2018 & Jan 2018 & \\
\hline IQR & July 2017- Sep 2018 & June 2017- Oct 2018 & July 2017- Aug 2018 & \\
\hline Min - Max & Jan 2017 - Feb 2019 & Jan 2017 - Feb 2019 & Jan 2017 - Feb 2019 & \\
\hline Age, years & & & & 0.602 \\
\hline Median & 8.5 & 10 & 8 & \\
\hline IQR & $3-14$ & $2-14$ & $3-14$ & \\
\hline Min - Max & $0-22$ & $0-19$ & $0-22$ & \\
\hline Mean \pm SD & $8.6 \pm 5.6$ & $8.9 \pm 5.9$ & $8.9 \pm 5.4$ & \\
\hline Age groups, N (\%) & & & & 0.070 \\
\hline $0-3 y$ & $57(28)$ & $25(30)$ & $32(27)$ & \\
\hline $4-6 y$ & $22(11)$ & $6(7)$ & $16(13)$ & \\
\hline $7-10 y$ & $42(21)$ & $13(16)$ & $29(24)$ & \\
\hline $11-13 y$ & $24(12)$ & $14(17)$ & $10(8)$ & \\
\hline $14-16 y$ & $44(22)$ & $15(18)$ & $29(24)$ & \\
\hline $17-22 y$ & $13(6)$ & $9(11)$ & $4(3)$ & \\
\hline Diagnosis, N (\%) & & & & 0.052 \\
\hline Leukaemia & $69(34)$ & $18(22)$ & $51(43)$ & \\
\hline Lymphoma & $26(13)$ & $13(16)$ & $13(11)$ & \\
\hline Solid tumour & $70(35)$ & $35(43)$ & $35(29)$ & \\
\hline MDS & $5(3)$ & $1(1)$ & $4(3)$ & \\
\hline Non-malignancies & $28(14)$ & $13(16)$ & $15(13)$ & \\
\hline Others & $4(2)$ & $2(2)$ & $2(2)$ & \\
\hline Puberty, N (\%) & & & & 0.134 \\
\hline Pre-pubertal & $132(65)$ & $48(59)$ & $84(70)$ & \\
\hline Pubertal & $4(2)$ & $1(1)$ & $3(3)$ & \\
\hline Post-pubertal & $66(33)$ & $33(40)$ & $33(28)$ & \\
\hline
\end{tabular}

$F P$, fertility preservation; IQR interquartile range; Min, minimum; Max, maximum; MDS, myelodysplastic syndrome
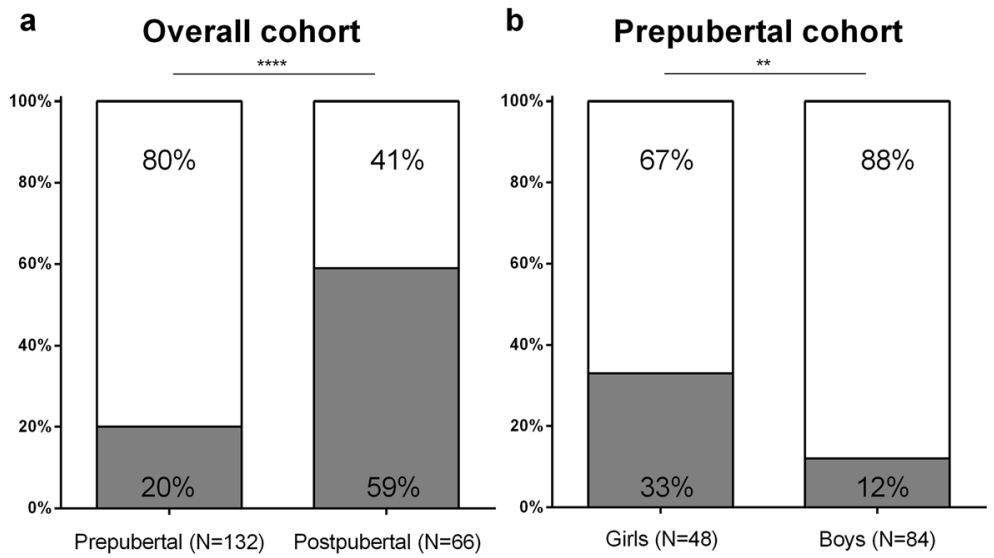

c Postpubertal cohort

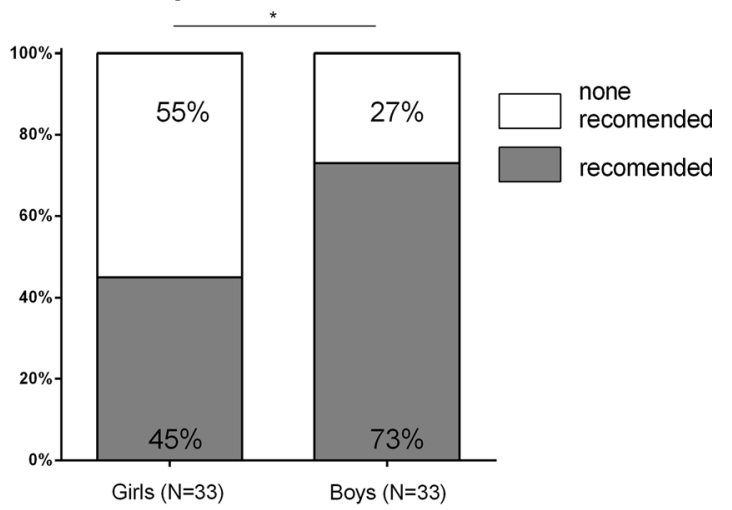

Fig. 2 Recommendations of fertility-preserving measures with regard to pubertal status and sex. For better clarity, we excluded peri-pubertal patients ( $N=1$ in girls, $N=3$ in boys). Significant differences between groups were assessed by Fisher's exact test. Differences were considered significant for $P<0.05$ (*), $P<0.01$ (**), and $P<0.001$ (***). The light color indicates that no procedure was recommended, and the dark area indicates that a fertility measure was recommended 
Prepubertal female patients **

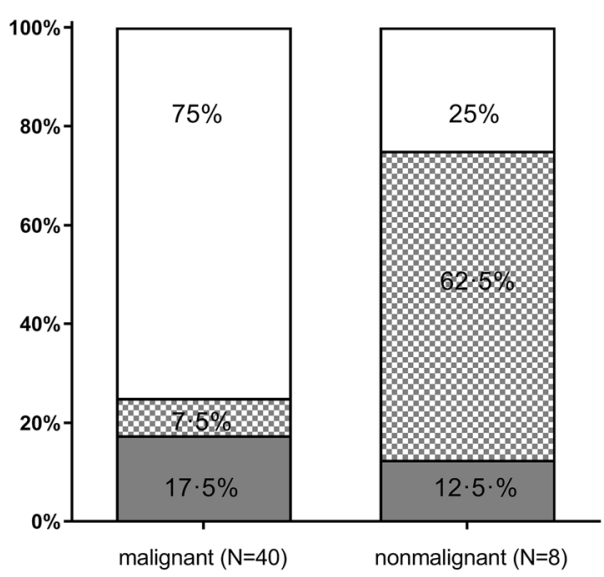

Postpubertal female patients

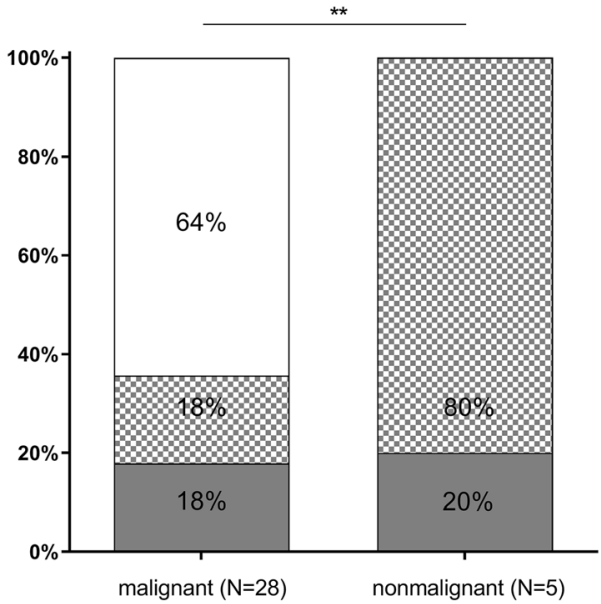

Fig. 3 Results of the counseling process: recommended and performed vs. non performed fertility-preserving procedures in prepubertal vs postpubertal female and male patients, separately examining the malignant and non-malignant cohorts. Significant differences between groups were assessed by Fisher's exact test. Differences were considered

\section{Discussion}

We present the first data following the initiation and implementation of fertility counseling in our single pediatric hematology/oncology, stem cell transplantation and immunology center in a situation where the costs of FP procedures not yet covered by the national health system in Germany. Before this structured process was started, there was no standardized fertility counseling for patients, and the discussion of the gonadotoxic side effects of cancer treatment formed only part of the general information before seeking informed consent concerning diagnosis and treatment. Despite a broader awareness by the public and the legal chances of financial support for fertilitypreserving measures, many pediatric hemato-oncology departments are experiencing a similar situation. After the
Prepubertal male patients
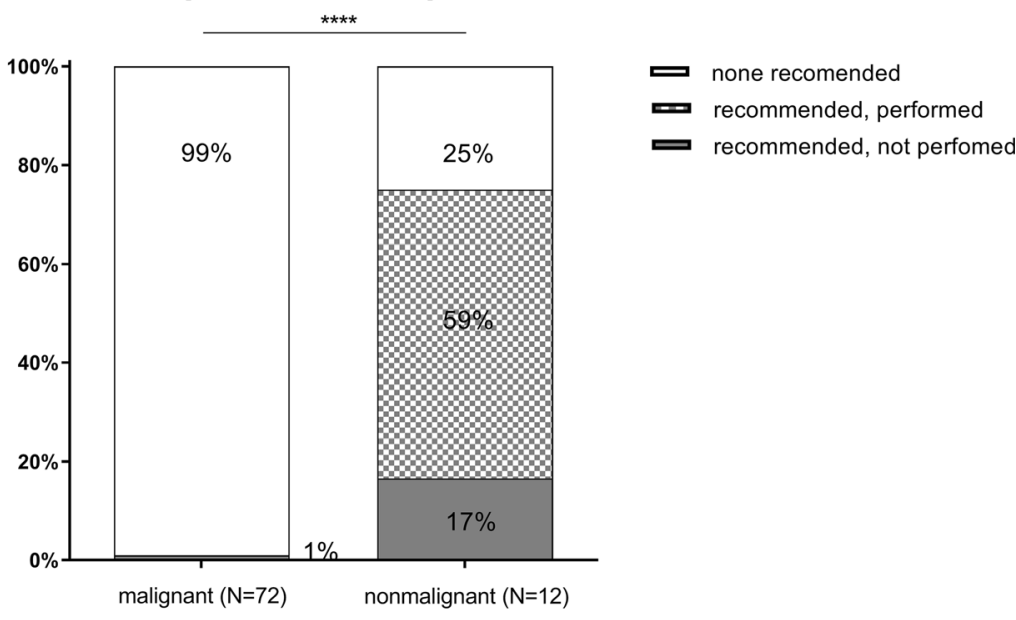

Postpubertal male patients
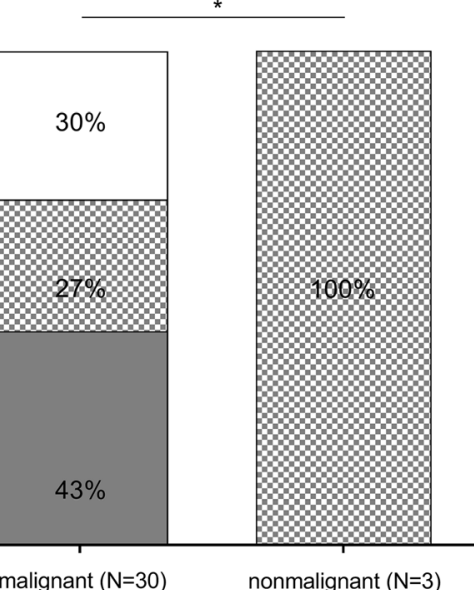

significant for $P<0.05$ (*), $P<0.01$ (**), $P<0.001$ (***), and $P<0.0001$ $(* * * *)$. Light color indicates that no measure was recommended, and squares indicate that measures were recommended and performed. Dark color represents a recommended measure that was not performed

structuring processes and implementation of a pattern for fertility counseling by the team using an inhouse standard procedure, it was possible to counsel approximately $90 \%$ of the newly diagnosed oncological patients and $100 \%$ of the patients undergoing HSCT according to our inhouse standard procedure for FP counseling. Patients not counseled included those initially admitted to the Intensive care unit due to a severe medical condition and those from other countries visiting our department only for portions of their treatment. The attending medical team always consulted with the counseling physician if fertility counseling was felt to be of benefit for relapsed patients and their family.

Recommendations regarding FP procedures were well accepted by the patients and their families. For prepubertal patients, especially in the $0-3$ year age group, we rarely recommended an invasive procedure. This approach 
will change in Germany in the coming years. After the implementation of severe combined immunodeficiency (SCID) screening in Germany in 2019, we recorded a number of very young patients $(<1$ year) who qualified for HSCT and thus potentially for fertility-preserving measures.

For most patients in the $0-3$ year age group with malignant diseases, FP procedure was not recommended due to the low risk of gonadotoxicity of the treatment or a high risk of contamination of the gonadal tissue with malignant cells. The retransplantation of cryopreserved immature gonadal tissue is still experimental, and there is a high risk of contamination with malignant cells in leukemia and certain solid tumors [20]. Further research is needed to determine possibilities in addressing potentially contaminated tissue, such as the in vitro maturation of immature oocytes [26]. Within the multidisciplinary team, we decided at the beginning of our project not to recommend tissue cryopreservation in these cases due to the highly experimental status of the procedure and the fact that health insurance in our country does not cover cryopreservation. Taking these points together, we decided that starting the implementation of the fertility counseling with the highest consensus from the team was important. Within the team, we agreed on the processes for reevaluating this decision and the consequences of our in-house standard procedure on a regular basis with the opportunity to change indications for invasive procedures in case of new research data and/or changes in coverage of the costs by the national health insurance.

Nevertheless, gonadal tissue collection from patients with leukemia can be considered, in view of future developments, for in vitro maturation and subsequent in vitro fertilization, and we offered it to families at their request. Notably, however, none decided to undergo the procedure.

In the subgroup of very young children, included patients with abdominal tumors (hepatoblastomas, neuroblastomas, nephroblastomas), abdominal surgery for ovarian tissue harvesting was thought to add potential complications. In some cases, the children were already critically ill; thus, we agreed with the parents not to place the child at further risk with an invasive procedure.

For prepubertal male patients, there is only the possibility of cryopreserving immature testicular tissue. Due to the experimental nature of the procedure and the risk for malignant cell infiltration, we recommend it only in cases with a high risk of gonadotoxicity for nonmalignant diseases (see the discussion above concerning contamination of gonadal tissue with malignant cells), almost exclusively for patients facing HSCT. The tissue was sent to the tissue bank of the University of Muenster as part of the FP project Androprotect [27]. In these cases, the percentage of patients who accepted undergoing a recommended measure was remarkably high (75\%) despite its experimental nature. Reasons not to perform a recommended and patient-approved procedure in older patients were associated either with worsening of the patient's medical condition or personal reasons of the patients. Only in rare cases were logistical reasons involved, e.g., timing of invasive procedures without a delay in initiating chemotherapy. Strikingly, the acceptance for sperm cryopreservation was $100 \%$ in the nonmalignant teenage cohort, while that in the malignant group was quite low. The reasons and implications of this difference are currently under investigation in our institution. Though the numbers of our cohort are still small (e.g., $N=3$ in the non-malignant male subgroup), this experience is consistent with other studies investigating the use of sperm cryopreservation in young adults [28, 29]. However, the majority of these patients in this study chose not to bank sperm or were not offered the opportunity. The authors discus a range of factors such as time, emotional state, patient age, disease stage, institutional practices, and cost all influence whether banking is offered to patients and taken up. Although the possibility for FP exists in our center, a detailed discussion took place and the costs of cryopreservation were covered for a certain period of time, only a few patients took advantage of the possibility. We speculate as well that this was due to the eminent irritation and distress of a newly diagnosed oncological disease. In these cases, we need to find ways to help teenaged male patients understand the importance of cryopreserving material and thus undergo sperm cryopreservation. Biopsy of the postpubertal testis is a feasible method if it is not possible to produce sperm spontaneously. For patients who did not succeed in producing sperm in sufficient number or quality, we discussed this method with the patient/family. In most cases, we agreed not to use this procedure due to its invasive nature (in addition to the need for anesthesia) and the possible delay of treatment by planning this procedure. It would be interesting to determine if more young men with malignant disease would agree to cryopreserve their sperm as part of a more distant procedure with less personal involvement.

Cryopreservation of oocytes in female patients is an established method of FP [30]. However, in our cohort, only one patient underwent this procedure. Cryopreservation of unfertilized oocytes requires 12-14 days of hormonal stimulation. Our oncological team agreed on a tight time frame for FP procedures, with a maximum delay of 7 days for the start of chemotherapy. There is no clear evidence regarding the degree to which different delays negatively influence the outcome [31]; thus, decisions such as these needs to be reevaluated on a regular basis. For patients with nonmalignant diseases such as thalassemia major, oocyte cryopreservation is 
a feasible method [32]. Patients with sickle cell disease are at risk of a sickle cell crisis triggered by hormonal stimulation; therefore, oocyte cryopreservation is generally not recommended for these patients [33].

\section{Implications of findings}

An in-house standard procedure for fertility-preserving procedures eased the implementation of fertility counseling at our institution and provided consistent yet individualized counseling for patients facing gonadotoxic treatment for either malignant or nonmalignant disease.

Other institutions have been able to show that after the structured establishment of fertility counseling, significantly more patients underwent FP procedures [34]. However, in a recent survey of FP counseling, even in EBMT settings for pediatric HSCT with a high risk of gonadotoxicity, less than half of the patients received FP counseling, and less than one third underwent FP procedures [35]. This demonstrates the need for broad FP programs. Our intention in establishing an in-house standard procedure was to enable comprehensive consultation for all pediatric patients with gonadotoxic risk in our pediatric clinic. In contrast to departments that care for adult patients facing gonadotoxic therapy, efforts to provide comprehensive counseling for children and their families remain in the early stages in many countries [7]. In-house standard procedures with indication lists for the fertility counseling of young patients based on a common consensus of the caring team and on recent guidelines would, in our view, simplify implementation considerably and simultaneously create awareness of the FP possibilities prior to gonadotoxic therapy. Meanwhile, an in-house standard procedure would help in determining which pediatric patients FP measures are indicated without delaying the start of therapy or imposing an additional risk through invasive measures for the child.

The implementation of fertility counseling using an inhouse indication list as a standard procedure could potentially be adapted first in national and later international settings to allow a more standardized handling of the counseling while allowing a consensus regarding the counseling within the multiprofessional team to be formed. However, this study describes the results of a single center experience, and adaptation through other institutions is pending to show that it is feasible to implement counseling for FP with an in-house standard operating procedure (SOP) according to recent FP guidelines. Ongoing evaluation of the counseling process should constantly improve it in terms of the needs of the patients and families.

In times of limited medical staff and time resources, it is important to ensure adequate scheduling for fertility counseling within the first days of oncological diagnosis. Therefore, additional funding sources are needed. In our case, counseling was enabled through the funding of a parents' association. In our institution, we have a multidisciplinary team composed of members of all departments involved in FP. There is effective cooperation with the departments of gynecology/reproductive medicine, andrology, pediatric surgery, and psychology. Within this network, it is possible to ensure quick and individual discussions of newly diagnosed patients and the realization of FP procedures if indicated. A multidisciplinary team that involves strong coordination and collaboration in the abovementioned fields is essential to guarantee FP for pediatric patients [36].

Official guidelines and recommendations (AWMF, ASCO, EBMT, and ARSM) represent the current medical opinion regarding FP. As they rely on former and recent data, it is important to assure ongoing discussion of recent research results in a young field such as FP for gonadotoxic therapy. Furthermore, ethical questions regarding how to counsel patients with genetic disorders as well as patients with disabilities are emerging issues that require discussion.

Acknowledgements We thank the parents' association "Hilfe für Krebskranke Kinder Frankfurt e.V.” for funding the project.

Author contribution $\mathrm{AB}$ and $\mathrm{AJ}$ counseled the patients; $\mathrm{NS}, \mathrm{HF}$, and $\mathrm{FO}$ counseled and treated the patients; $\mathrm{AB}$ and $\mathrm{AJ}$ collected patient data; $\mathrm{AB}$ and $\mathrm{AJ}$ wrote the manuscript; $\mathrm{ES}$ and $\mathrm{AB}$ performed data analysis and generated the graphics; $\mathrm{AB}, \mathrm{AJ}, \mathrm{NS}, \mathrm{HF}, \mathrm{FO}, \mathrm{PB}$, and TK discussed the draft of the manuscript.

Funding Open Access funding enabled and organized by Projekt DEAL. Funding was available through a parents' association, including for a physician scientist position (50\%), as the German health insurance system did not yet cover the costs as mentioned in the manuscript. Otherwise, funding was used to support the families covering the costs of the fertilitypreserving procedures.

Data availability All data related to this study are available on request.

Code availability Not applicable

\section{Declaration}

Conflict of interest The authors declare no competing interests.

Open Access This article is licensed under a Creative Commons Attribution 4.0 International License, which permits use, sharing, adaptation, distribution and reproduction in any medium or format, as long as you give appropriate credit to the original author(s) and the source, provide a link to the Creative Commons licence, and indicate if changes were made. The images or other third party material in this article are included in the article's Creative Commons licence, unless indicated otherwise in a credit line to the material. If material is not included in the article's Creative Commons licence and your intended use is not permitted by statutory regulation or exceeds the permitted use, you will need to obtain 
permission directly from the copyright holder. To view a copy of this licence, visit http://creativecommons.org/licenses/by/4.0/.

\section{References}

1. Leisenring WM, Mertens AC, Armstrong GT, Stovall MA, Neglia JP, Lanctot JQ, Boice JD Jr, Whitton JA, Yasui Y (2009) Pediatric cancer survivorship research: experience of the childhood cancer survivor study. J Clin Oncol 27:2319-2327

2. European Society for Blood and Marrow Transplantation. Annual Report, 2017. EBMT: Leiden, The Netherlands, 2017. https:// www.ebmt.org/ebmt/documents/ebmt-annual-report-2017

3. Zynda A, Reinmuth S, Pfitzer C, Hohmann C, Keil T, BorgmannStaudt A (2012) Childhood leukemia and its impact on graduation and having children: results from a national survey. Leuk Lymphoma 53:2419-2422

4. Reinmuth S, Hohmann C, Rendtorff R, Balcerek M, Holzhausen S, Müller A, Henze G, Keil T, Borgmann-Staudt A (2013) Impact of chemotherapy and radiotherapy in childhood on fertility in adulthood: the FeCt-survey of childhood cancer survivors in Germany. $\mathrm{J}$ Cancer Res Clin Oncol 139:2071-2078

5. Chow EJ, Stratton KL, Leisenring WM, Oeffinger KC, Sklar CA, Donaldson SS, Ginsberg JP, Kenney LB, Levine JM, Robison LL, Shnorhavorian M, Stovall M, Armstrong GT, Green DM (2016) Pregnancy after chemotherapy in male and female survivors of childhood cancer treated between 1970 and 1999: a report from the childhood cancer survivor study cohort. Lancet Oncol 17: 567-576

6. Pfitzer C, Orawa H, Balcerek M, Langer T, Dirksen U, Keslova P, Zubarovskaya N, Schuster FR, Jarisch A, Strauss G, BorgmannStaudt A (2015) Dynamics of fertility impairment and recovery after allogeneic hematopoietic stem cell transplantation in childhood and adolescence: results from a longitudinal study. J Cancer Res Clin Oncol 141:135-142

7. Dalle JH, Lucchini G, Balduzzi A, Ifversen M, Jahnukainen K, Macklon KT, Ahler A, Jarisch A, Ansari M, Beohou E, Bresters D, Corbacioglu S, Dalissier A, Diaz de Heredia Rubio C, Diesch T, Gibson B, Klingebiel T, Lankester A, Lawitschka A et al (2017) State-of-the-art fertility preservation in children and adolescents undergoing hematopoietic stem cell transplantation: a report on the expert meeting of the Pediatric Diseases Working Party (PDWP) of the European Society for Blood and Marrow Transplantation (EBMT) in Baden, Austria, 29-30 September 2015. Bone Marrow Transplant 52:1029-1035

8. https://www.bundesgesundheitsministerium.de/terminservice-undversorgungsgesetz.html (as of 11/17/19)

9. AWMF. S1 Leitlinie 025/034 “Beeinträchtigung der Gonadenfunktion nach Chemo- und Strahlentherapie im Kindersund Jugendalter. 2015. https://www.awmf.org/uploads/tx szleitlinien/025-034 1 S1 Beeintr\%c3\%a4chtigung Gonadenfunktion nach Krebs im Kindesalter 2015-03.pdf.

10. Barlevy D, Wangmo T, Elger BS, Ravitsky V (2016) Attitudes, beliefs, and trends regarding adolescent oncofertility discussions: a systematic literature review. J Adolesc Young Adult Oncol 5: $119-134$

11. Informed Consent in DecisionMaking in Pediatric Practice COMMITTEE ON BIOETHICS

12. Picton HM, Wyns C, Anderson RA, Goossens E, Jahnukainen K, Kliesch S, Mitchell RT, Pennings G, Rives N, Tournaye H, van Pelt A, Eichenlaub-Ritter U, Schlatt S, ESHRE Task Force On Fertility Preservation In Severe Diseases (2015) A European perspective on testicular tissue cryopreservation for fertility preservation in prepubertal and adolescent boys. Hum Reprod 30:2463-2475
13. Stukenborg JB, Alves-Lopes JP, Kurek M et al (2018) Spermatogonial quantity in human prepubertal testicular tissue collected for fertility preservation prior to potentially sterilizing therapy. Hum Reprod 33:1677-1683

14. Fayomi AP, Peters K, Sukhwani M, Valli-Pulaski H, Shetty G, Meistrich ML, Houser L, Robertson N, Roberts V, Ramsey C, Hanna C, Hennebold JD, Dobrinski I, Orwig KE. Autologous grafting of cryopreserved prepubertal rhesus testis produces sperm and offspring. Science. 2019 Mar 22;363(6433):1314-1319. doi: 10.1126/science.aav2914. Erratum in: Science. 2019 Apr 5; 364(6435):

15. Pinelli S, Basile S (2018) Fertility preservation: current and future perspectives for oncologic patients at risk for iatrogenic premature ovarian insufficiency. Biomed Res Int 2018:6465903

16. Jensen AK, Macklon KT, Fedder J, Ernst E, Humaidan P, Andersen CY (2017) 86 successful births and 9 ongoing pregnancies worldwide in women transplanted with frozen-thawed ovarian tissue: focus on birth and perinatal outcome in 40 of these children. $\mathrm{J}$ Assist Reprod Genet 34:325-336

17. Fertility preservation in patients undergoing gonadotoxic therapy or gonadectomy: a committee opinion. Practice Committee of the American Society for Reproductive Medicine. Electronic address: asrm@asrm.org. Fertil Steril. 2019 Dec;112(6):1022-1033. doi: 10.1016/j.fertnstert.2019.09.013.

18. Matthews SJ, Picton H, Ernst E, Andersen CY (2018) Successful pregnancy in a woman previously suffering from beta-thalassemia following transplantation of ovarian tissue cryopreserved before puberty. Minerva Ginecol 70:432-435.27

19. Jadoul P, Dolmans MM, Donnez J (2010) Fertility preservation in girls during childhood: is it feasible, efficient and safe and to whom should it be proposed? Hum Reprod Update 16:617-630

20. Dolmans MM, Luyckx V, Donnez J, Andersen CY, Greve T (2013) Risk of transferring malignant cells with transplanted frozenthawed ovarian tissue. Fertil Steril 99:1514-1522

21. Asadi-Azarbaijani B, Sheikhi M, Nurmio M, Tinkanen H, Juvonen V, Dunkel L, Hovatta O, Oskam IC, Jahnukainen K (2016) Minimal residual disease of leukemia and the quality of cryopreserved human ovarian tissue in vitro. Leuk Lymphoma. 57(3):700 707

22. Meirow D, Hardan I, Dor J, Fridman E, Elizur S, Ra'anani H, Slyusarevsky E, Amariglio N, Schiff E, Rechavi G, Nagler A, Ben Yehuda D. Searching for evidence of disease and malignant cell contamination in ovarian tissue stored from hematologic cancer patients. Hum Reprod. 2008 May;23(5):1007-13.

23. Dittrich R, Kliesch S, Schüring A, Balcerek M, Baston-Büst DM, Beck R, Beckmann MW, Behringer K, Borgmann-Staudt A, Cremer W, Denzer C, Diemer T, Dorn A, Fehm T, Gaase R, Germeyer A, Geue K, Ghadjar P, Goeckenjan M et al (2018) Fertility preservation for patients with malignant disease. Guideline of the DGGG, DGU and DGRM (S2k-Level, AWMF Registry No. 015/082, November 2017) - recommendations and statements for girls and women. Geburtshilfe Frauenheilkd 78: $567-584$

24. Loren AW, Mangu PB, Beck LN, Brennan L, Magdalinski AJ, Partridge AH, Quinn G, Wallace WH, Oktay K, American Society of Clinical Oncology (2013) Fertility preservation for patients with cancer: American society of clinical oncology clinical practice guideline update. J Clin Oncol 31:2500-2510

25. Balduzzi A, Dalle JH, Jahnukainen K et al (2017) Fertility preservation issues in pediatric hematopoietic stem cell transplantation: practical approaches from the consensus of the pediatric diseases working party of the EBMT and the international BFM study group. Bone Marrow Transplant 52:1406-1415

26. Current state and future possibilities of ovarian tissue transplantation Seido Takae, Nao Suzuki. Reprod Med Bio. 2019 Apr 8;18(3): 217-224. 
27. https:/www.medizin.uni-muenster.de/cera/ (as of 11/17/19)

28. Sonnenburg DW, Brames MJ, Case-Eads S, Einhorn LH (2015 Sep) Utilization of sperm banking and barriers to its use in testicular cancer patients. Support Care Cancer. 23(9):2763-2768

29. Klosky JL, Randolph ME, Navid F, Gamble HL, Spunt SL, Metzger ML, Daw N, Morris EB, Hudson MM (2009) Sperm cryopreservation practices among adolescent cancer patients at risk for infertility. Pediatr Hematol Oncol 26(4):252-260

30. Rienzi L, Gracia C, Maggiulli R, LaBarbera A, Kaser DJ, Ubaldi FM, Vanderpoel S, Racowsky C (2017) Oocyte, embryo and blastocyst cryopreservation in ART: systematic review and metaanalysis comparing slow-freezing versus vitrification to produce evidence for the development of global guidance. Hum Reprod Update 23:139-155

31. Baker JM, To T, Beyene J, Zagorski B, Greenberg ML, Sung L (2014) Influence of length of time to diagnosis and treatment on the survival of children with acute lymphoblastic leukemia: a population-based study. Leuk Res 38:204-209

32. Carlberg KT, Singer ST, Vichinsky EP (2018) Fertility and pregnancy in women with transfusion-dependent thalassemia. Hematol Oncol Clin North Am 32:297-315

33. Matthews M, Pollack R (2017) Acute pain crisis in a patient with sickle cell disease undergoing ovarian simulation for fertility preservation prior to curative stem cell transplantation: case report and literature review. J Assist Reprod Genet 34:1445-1448

34. Carlson CA, Kolon TF, Mattei P, Hobbie W, Gracia CR, Ogle S, Ginsberg JP (2017 Nov) Developing a Hospital-Wide Fertility Preservation Service for Pediatric and Young Adult Patients. J Adolesc Health. 61(5):571-576

35. Diesch T, Rovo A, von der Weid N, Faraci M, Pillon M, Dalissier A, Dalle JH, Bader P. Fertility preservation practices in pediatric and adolescent cancer patients undergoing HSCT in Europe: a population-based survey. Bone Marrow Transplant. 2017 Jul;52(7):1022-1028. doi: 10.1038/bmt.2016.363. Epub 2017 Jan 23.PMID: 28112743

36. Salama M, Anazodo A, Woodruff TK (2019 Nov 1) Preserving fertility in female patients with hematological malignancies: a multidisciplinary oncofertility approach. Ann Oncol. 30(11):17601775. https://doi.org/10.1093/annonc/mdz284

Publisher's note Springer Nature remains neutral with regard to jurisdictional claims in published maps and institutional affiliations. 